\title{
Synthesis of Axially Chiral Vinyl Arenes by Palladium(0) Catalysis
}

Gategory

Metal-Catalyzed

Asymmetric

Synthesis and

Stereoselective

Reactions

\section{Key words}

palladium catalysis

vinyl arenes

carbenes

phosphine-olefin

ligands

Selected examples:<smiles></smiles>

$\mathrm{Pd}(\mathrm{OAc})_{2}(10 \mathrm{~mol} \%)$<smiles>O=P([Al])([Al])c1ccc2cnccc2c1Br</smiles>
ligand $(20 \mathrm{~mol} \%)$ $t$-BuOLi (2.5 equiv)

1,4-dioxane, $50^{\circ} \mathrm{C}, 24 \mathrm{~h}$ (0.2 mmol scale)
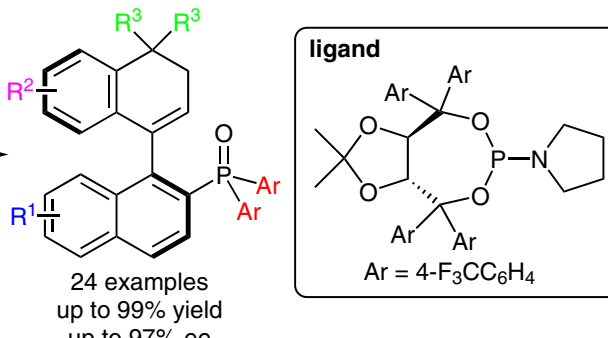

up to $97 \%$ ee
$\mathrm{Ar}=4-\mathrm{F}_{3} \mathrm{CC}_{6} \mathrm{H}_{4}$
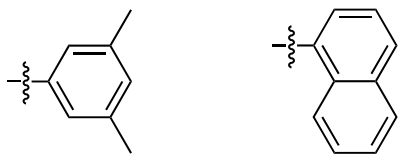

$55 \%$ yield, $93 \%$ ee

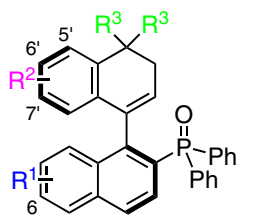

- $\mathrm{R}^{1}=\mathrm{R}^{3}=\mathrm{H}$

$\mathrm{R}^{2}=7^{\prime}-\mathrm{Cl}, 99 \%$ yield, $85 \%$ ee

$\mathrm{R}^{2}=6^{\prime}-\mathrm{Cl}, 86 \%$ yield, $87 \%$ ee

$R^{2}=5^{1}-\mathrm{OMe}, 99 \%$ yield, $95 \%$ ee

- $\mathrm{R}^{1}=\mathrm{R}^{2}=\mathrm{H}$

$\mathrm{R}^{3}=\mathrm{Me}, 65 \%$ yield, $91 \%$ ee $(48 \mathrm{~h})$

$\mathrm{R}^{3}=-\mathrm{CH}_{2} \mathrm{CH}_{2} \mathrm{CH}_{2} \mathrm{CH}_{2}-, 40 \%$ yield, $91 \%$ ee $(48 \mathrm{~h})$ - $\mathrm{R}^{2}=\mathrm{R}^{3}=\mathrm{H}$

$\mathrm{R}^{1}=6-\mathrm{CO}_{2} i-\mathrm{Pr}, 84 \%$ yield, $87 \%$ ee $(48 \mathrm{~h})$

Application as chiral ligand:

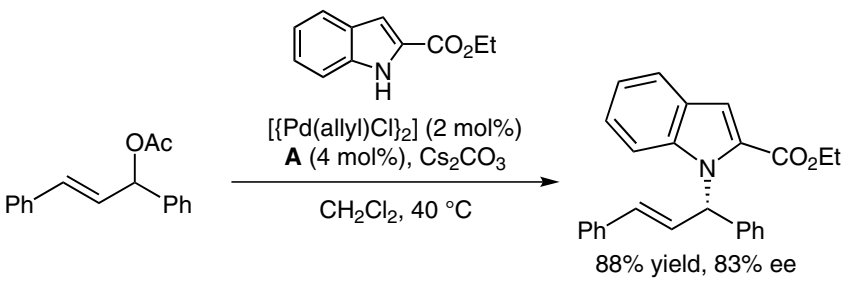

$88 \%$ yield, $83 \%$ ee

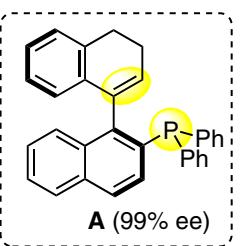

(a)

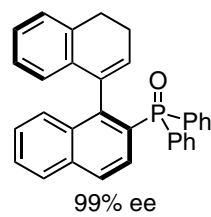

(a) $(\mathrm{COCl})_{2}, \mathrm{CH}_{2} \mathrm{Cl}_{2}$, r.t. then $\mathrm{LiAlH}_{4}, \mathrm{THF}, 0^{\circ} \mathrm{C}$ $95 \%$ yield
Significance: The authors report the highly enantioselective synthesis of various vinyl arenes via a palladium-catalyzed cross-coupling reaction. The vinyl-arene compounds were easily transformed into binaphthyl skeletons or phosphine compounds. The authors also demonstrate an application of the product as a chiral phosphine-olefin ligand in an allylic substitution reaction.

SYNFACTS Contributors: Mark Lautens, Kosuke Yamamoto Synfacts 2016, 12(3), $0261 \quad$ Published online: 16.02.2016 DOI: 10.1055/s-0035-1561744; Reg-No.: L00716SF
Comment: The reported coupling reaction involves oxidative addition of palladium(0) to bromoarenes, reaction with the diazo compound to generate a palladium-carbene species, migratory insertion and $\beta$-hydride elimination. A broad substrate scope was demonstrated and a range of chiral vinyl arenes were obtained. These products might be a new class of chiral phosphine-olefin ligands. 